\title{
Concepciones sobre sistemas de ecuaciones lineales de $3 \times 2$ con solución vacía: un estudio exploratorio con estudiantes universitarios
}

\author{
Andrea D. Cárcamo, Claudio E. Fuentealba y Felipe J. Tauler \\ Facultad de Ciencias de la Ingeniería, Centro de Docencia de Ciencias Básicas para Ingeniería, Universidad Austral de \\ Chile, General Lagos 2086, Campus Miraflores, Valdivia-Chile (corre-e: andrea.carcamo@uach.cl; cfuentealba@uach.cl; \\ felipe.tauler@uach.cl)
}

Recibido Jul. 2, 2020; Aceptado Ago. 28, 2020; Versión final Oct. 13, 2020, Publicado Feb. 2021

\begin{abstract}
Resumen
El objetivo de este estudio exploratorio es identificar las concepciones que muestran estudiantes universitarios de primer año al enfrentarse a preguntas que implican resolver sistemas de ecuaciones lineales (SEL) de 3x2 con solución vacía. El instrumento de recolección de datos fue una evaluación diagnóstica aplicada a 60 estudiantes que cursaban álgebra lineal e iniciaban el estudio de SEL. Al analizar los datos recopilados sobre la solución de los estudiantes de un SEL de 3x2 con solución vacía, se identificaron tres concepciones: 1) el SEL de $3 \times 2$ no tiene solución, 2) el SEL de 3x2 tiene tres soluciones (pues las rectas se interceptan en 3 puntos o hay 3 intercepciones) y 3) el SEL de 3x2 tiene solución única. Se concluye que estas concepciones erróneas permiten inferir que los estudiantes no comprenden el significado de la solución de un SEL y, por esta razón, presentan inconvenientes para resolver correctamente un SEL presentado en forma gráfica o analítica.
\end{abstract}

\section{Conceptions about systems of linear equations of $3 \times 2$ with an empty solution: an exploratory study with university students}

\begin{abstract}
The objective of the present study is to identify the conceptions that first-year undergraduate students have when faced with questions that involve solving a 3x2 systems of linear equations (SLE) with an empty solution. The data collection instrument was a diagnostic evaluation applied to 60 students enrolled in linear algebra who were starting to study SLE. The results showed that students had three different conceptions about the

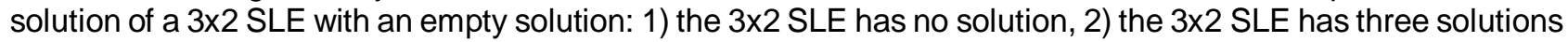
because lines intersect at 3 points or there are 3 interceptions, and 3) the $3 \times 2$ SLE has one unique solution. It is concluded that these misconceptions suggest that students lack understanding of the meaning of SLE solutions and that, for this reason, students have difficulties solving SLEs that are presented graphically or analytically.
\end{abstract}

Keywords: systems of linear equations; linear algebra; university students; empty solution 


\section{INTRODUCCIÓN}

Los sistemas de ecuaciones lineales (SEL) constituyen un tema de estudio en diferentes niveles escolares y, generalmente, a partir del nivel secundario es obligatorio en muchos países del mundo. Los SEL se consideran fundamentales por sus diversas aplicaciones tanto en el campo de la ingeniería como de las ciencias sociales (Possani et al., 2010) y por su importancia como contenido base para estudios posteriores en el área de matemáticas como, por ejemplo, el curso de Álgebra Lineal (Zandieh y Andrews-Larson, 2019) en donde se encuentran con sofisticadas definiciones y teoremas, que en muchas ocasiones deben demostrar (Roa-Fuentes y Parraguez, 2017). A pesar de esto, los SEL no han sido estudiados ampliamente por los especialistas en Educación Matemática (Oktaç, 2018). Desde este contexto, nuestro interés es aportar a la investigación sobre este contenido matemático, a nivel universitario, con la finalidad de proporcionar antecedentes que permitan desarrollar procesos de instrucción basados en evidencia empírica.

Los errores que los estudiantes tienen con los SEL, de acuerdo con Oktaç (2018), se relacionan estrechamente con generalizaciones que ellos hacen de sus estudios iniciales asociados a este contenido. Por ejemplo, en la enseñanza secundaria comienzan a desarrollar una concepción que asocia a los SEL con dos ecuaciones que contienen dos incógnitas y que se resuelven juntas para dar como resultado un par ordenado. Como consecuencia de esto, asocian la solución de un SEL con el par ordenado que obtuvieron al resolver dos ecuaciones simultáneamente y lo relacionan, geométricamente, con la intersección de dos rectas en el plano. La interpretación de una solución de un SEL, como un punto de intersección de dos rectas, funciona bien en el contexto de los SEL de $2 \times 2$. Sin embargo, se convierte en un obstáculo cuando este contexto cambia. Otro tipo de generalización que los estudiantes hacen es pensar que un SEL debe involucrar igual número de ecuaciones y de incógnitas. Por otra parte, Rodríguez, Mena, Mena, Vásquez y Del Valle (2019) concluyen que introducir los SEL $2 \times 2$ y $n \times 2$ como casos particulares, de situaciones más generales, conllevará a que los estudiantes tengan dificultad con pasar de la dimensión dos al caso general de dimensión finita, o la de extender el concepto de dependencia lineal del caso de dos al de más elementos en el conjunto considerado.

Las investigaciones sobre SEL de 3×2 reportan que los estudiantes presentan dificultades con su resolución. Por ejemplo, Almouloud y Bianchini (1996) identificaron que los estudiantes, a la hora de resolver un SEL de $3 \times 2$, creen que son necesarias solo dos ecuaciones e ignoran el hecho de que la solución del SEL debe satisfacer cada una de las ecuaciones. Recientemente, Peña (2019), en un estudio sobre las dificultades y errores relativos al concepto de solución de un SEL, detectó como error que, al resolver un SEL de 3x2, los estudiantes agrupan de dos en dos las ecuaciones, y así, obtienen como solución tres pares ordenados distintos. Además, otro error que identificó es que algunos estudiantes consideran como solución todos los puntos de corte entre las gráficas de las rectas que conforman el SEL, así como, las intersecciones de estas con los ejes coordenados, es decir, si se les presenta la gráfica de tres rectas de manera que se intercepten en tres puntos no colineales, el SEL tiene tres soluciones y otros, incluso, indican que tienen más soluciones, pues agregan los cortes con los ejes coordenados.

De acuerdo con los estudios sobre SEL y en particular, los que implican SEL de 3x2, los estudiantes presentan dificultades con estos. Por esta razón, nos interesa detectar las concepciones de los estudiantes en la resolución de este tipo de SEL para contribuir a corregir sus concepciones erróneas en la resolución de estos, si es que las tuvieran. Así, nuestro estudio exploratorio busca responder a la siguiente pregunta: ¿Cuáles con las concepciones previas de estudiantes universitarios de primer año cuando resuelven SEL de $3 \times 2$ que tienen solución vacía? De esta forma, el objetivo de este estudio es identificar las concepciones previas que muestran estudiantes universitarios de primer año cuando resuelven SEL de 3x2 que tienen solución vacía.

\section{OTROS ANTECEDENTES}

El marco teórico de este estudio exploratorio corresponde a los tres modos de pensamiento en Álgebra Lineal (Sierpinska, 2000): sintético-geométrico, analítico-aritmético y analítico-estructural. En el modo sintético (sintético-geométrico) los objetos matemáticos son dados directamente a la mente que luego, intenta describirlos. En tanto, en el modo analítico (analítico-aritmético y analítico-estructural) los objetos matemáticos son dados indirectamente, es decir, se construyen mediante las definiciones de las propiedades de sus elementos. Así, el modo sintético pertenece al modo práctico de pensar, y el analítico, al modo teórico de pensar (Sierpinska, 2000).

Cada uno de los tres modos de pensamiento en Álgebra Lineal usa un sistema específico de representaciones. El modo de pensamiento sintético-geométrico utiliza el lenguaje de las figuras geométricas (planos y líneas, intersecciones), así como sus representaciones gráficas convencionales. En tanto, el modo analíticoaritmético, comprende las figuras geométricas como conjuntos de n-tuplas de números que satisfacen determinadas condiciones y que se escriben, por ejemplo, en forma de sistemas de ecuaciones 0 
desigualdades. En este modo, los componentes numéricos de los objetos geométricos, como puntos o vectores, son importantes. Por ejemplo, un sistema de ecuaciones general se escribiría con todos los coeficientes explícitamente. Finalmente, el pensamiento analítico-estructural sintetiza los elementos algebraicos de las representaciones analíticas en totalidades estructurales. En este modo, por ejemplo, un sistema de ecuaciones general se escribiría en forma de matriz como $\mathrm{Ax}=\mathrm{b}$, o en forma vectorial (Sierpinska, 2000).

\section{METODOLOGÍA}

Este estudio se enmarca en una metodología de tipo cualitativa con carácter exploratorio y descriptivo (Maxwell, 1992) cuyo fin es identificar las concepciones previas de los estudiantes relativas a la solución de un SEL para, posteriormente, categorizarlas y describirlas.

\section{Participantes}

En este estudio, participaron 60 estudiantes de primer año de ingeniería de una universidad chilena que cursaban Álgebra Lineal. Estos participantes los denominaremos correlativamente desde E1, E2 hasta E60. Ellos, de acuerdo con las bases curriculares del Ministerio de Educación de Chile (MINEDUC, 2015), deben saber resolver sistemas de ecuaciones lineales (2x2) mediante representaciones gráficas y simbólicas.

\section{Instrumento de recolección de datos}

Con la finalidad de conocer las distintas concepciones de los estudiantes universitarios de primer año sobre los SEL de $3 \times 2$ con solución vacía, en esta investigación, se usó una evaluación diagnóstica que fue contestada individualmente, antes de que iniciaran el estudio formal de los SEL en el curso de Álgebra Lineal. Este instrumento constó de varias tareas que tenían como finalidad identificar las concepciones de los estudiantes sobre los SEL con dos incógnitas y con igual o diferente número de ecuaciones. Para el diseño y construcción del instrumento se consideraron los estudios previos sobre SEL reportados en la sección Introducción y antecedentes de este estudio.

\section{Análisis de datos}

El proceso de análisis de datos constó de tres fases. En la primera fase, dos investigadores del área de Educación Matemática y profesores de matemáticas en ejercicio, agruparon las respuestas de la evaluación diagnóstica como correctas, incorrectas o no responde. A partir de esta tipificación, se seleccionaron dos tareas para analizarlas porque tenían un mayor porcentaje de respuestas incorrectas $u$ omitidas. A estas tareas las denominaremos, en adelante, tarea 1 y tarea 2. La Tabla 1 presenta las frecuencias absolutas y porcentuales de respuestas correctas, incorrectas y omitidas de estas tareas de la evaluación diagnóstica (Fig. 1).

Tabla 1: Tipos de respuestas dadas a las tareas 1 y 2 por los estudiantes

\begin{tabular}{|l|c|c|c|c|c|c|}
\hline \multirow{2}{*}{ Frecuencia } & \multicolumn{5}{|c|}{ Tespuestas } \\
\cline { 2 - 7 } & \multicolumn{3}{|c|}{ Tarea 1 } & \multicolumn{3}{c|}{ Tarea 2 } \\
\cline { 2 - 7 } & Correcta & Incorrecta & Omitida & Correcta & Incorrecta & Omitida \\
\hline Absoluta & 15 & 38 & 7 & 23 & 36 & 1 \\
\hline Porcentual & $25 \%$ & $63 \%$ & $12 \%$ & $38 \%$ & $60 \%$ & $2 \%$ \\
\hline
\end{tabular}

La tarea 1 se construyó de manera similar a una reportada en Oktaç (2018) mientras que la tarea 2, se extrajo textual de esta misma investigación. Ambas tareas están relacionadas con los modos de pensamiento planteados por Sierpinska (2000) y se presentan en la Figura 1. Ambas preguntan por la solución de un SEL de $3 \times 2$ que tienen como solución el conjunto vacío. Sin embargo, se diferencian en que, en la tarea 1, se muestra la representación gráfica del SEL. En tanto, en la tarea 2, el SEL se encuentra expresado de forma analítica.

En la segunda fase, se transcribieron los protocolos escritos de los estudiantes, para las tareas 1 y 2 , en una matriz con la finalidad de conocer las distintas concepciones de los estudiantes sobre la resolución de un SEL de $3 \times 2$ con solución vacía. A continuación, las transcripciones se analizaron en profundidad, observando el proceso usado por el estudiante y la solución entregada. Finalmente, en la tercera fase, se identificaron y categorizaron las concepciones que fueron más frecuentes entre los estudiantes sobre la resolución de los SEL de $3 \times 2$ que tenían solución vacía. 


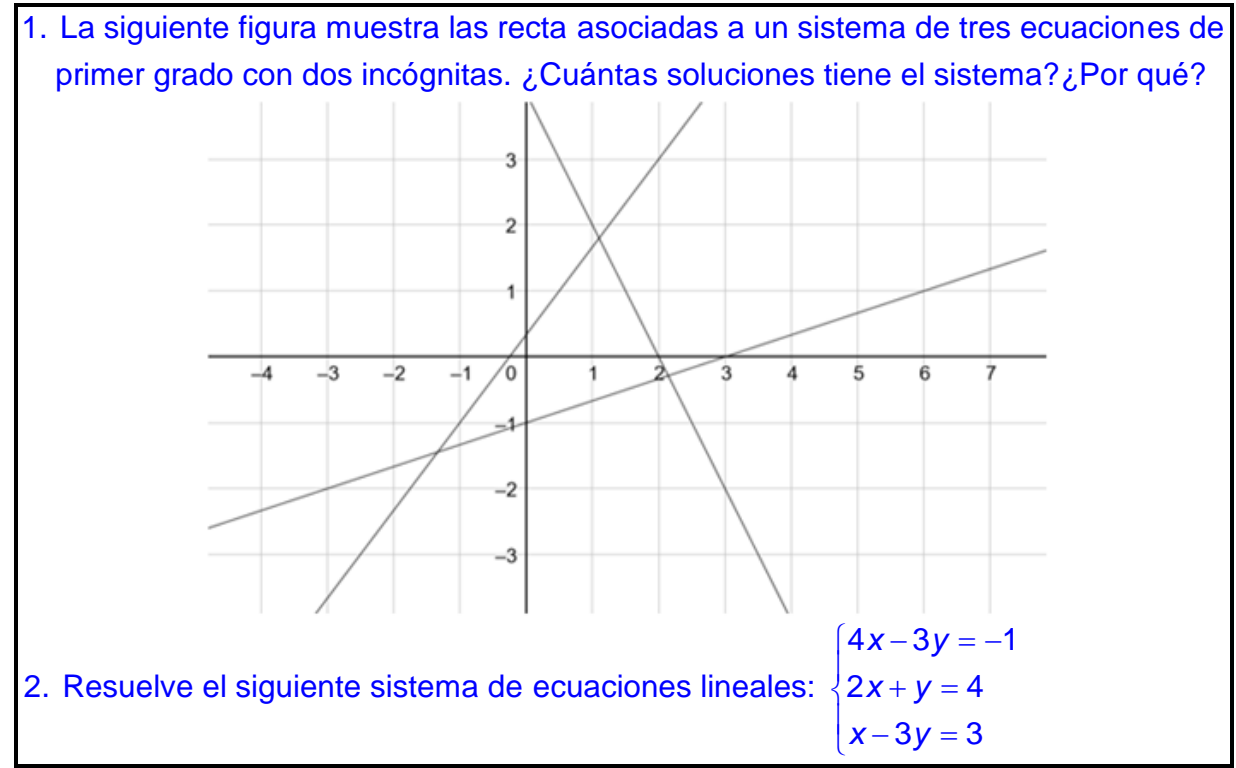

Fig. 1: Tareas de la evaluación diagnóstica sobre SEL de 3x2

\section{RESULTADOS}

El análisis de los datos recopilados en este estudio, permitió identificar tres concepciones de los estudiantes respecto a la resolución de un SEL de $3 \times 2$ que posee solución vacía, dos de las cuales son erróneas. Estas concepciones son: el SEL de $3 \times 2$ no tiene solución, el SEL de $3 \times 2$ tiene 3 soluciones (pues las rectas se interceptan en 3 puntos o hay 3 intercepciones) y el SEL de $3 \times 2$ tiene solución única. A continuación, describimos estas tres concepciones de los estudiantes a través sus protocolos escritos.

\section{EI SEL de 3x2 no tiene solución}

Al resolver el SEL de $3 \times 2$ de la tarea 1, los estudiantes que responden correctamente, escribieron que no tenía solución. En el caso de la tarea 2, los estudiantes que responden correctamente, la mayoría anotó que no tenía solución (un 75\%) mientras que los demás escribieron que tenía solución vacía. Los estudiantes que respondieron que el SEL de 3x2 de la tarea 1 (representado gráficamente) no tenía solución argumentaron que no había ningún punto común en el que las tres rectas se intersecaran. En la Tabla 2 se observan algunos ejemplos de este tipo de explicaciones de los estudiantes E33, E27 y E20. El estudiante E33 escribió "no tiene solución porque no existe un punto donde se intercepten las 3 rectas". Una respuesta similar anotó E27. En tanto, el estudiante E20 precisó que no tenía ninguna solución porque "no se interceptan las tres rectas en ningún punto en común". Las respuestas de estos estudiantes dan evidencia de que saben interpretar la representación gráfica de este tipo de SEL y a partir de esta, entregar la solución.

Tabla 2: Ejemplos de respuesta de los estudiantes que identificaron que el SEL de $3 \times 2$ de la Tarea 1 no tiene solución

\begin{tabular}{|l|l|}
\hline Estudiante & Fragmento de respuesta \\
\hline E33 & No tiene solución porque no existe un punto donde se intercepten las 3 rectas \\
\hline E27 & No tiene solución porque no hay punto donde se intercepten las 3 rectas \\
\hline E20 & Ninguna, ya que no se interceptan las tres rectas en ningún punto en común \\
\hline
\end{tabular}

Por otro lado, los estudiantes para responder que el SEL de $3 \times 2$ de la tarea 2 (representado analíticamente) no tenía solución, usaron en su mayoría, su conocimiento previo sobre método de sustitución o método de reducción. Solo dos estudiantes usaron el método de igualación. Por ejemplo, el estudiante E2 utilizó el método de sustitución. Como se observa en su respuesta escrita (Fig.2), E2 despejó la incógnita y de la segunda ecuación del SEL $(2 x+y=4)$ de lo que obtiene $y=4-2 x$. A continuación, sustituyó la incógnita $y$ en la primera ecuación del SEL y resolvió la ecuación lineal resultante de la que obtuvo $x=11 / 10$. Luego, reemplazó este valor de $x$ en la segunda ecuación, lo que le dio como resultado $y=9 / 5$. Finalmente, evaluó los valores de $x$ e $y$ obtenidos en las tres ecuaciones del SEL. A partir de esto, E2 concluyó que estos valores satisfacen solo dos de las tres ecuaciones del SEL, por lo que el sistema no posee solución. De su respuesta, podemos inferir que E2 sabe que para que un SEL tenga solución, esta última debe satisfacer todas las ecuaciones del SEL. 


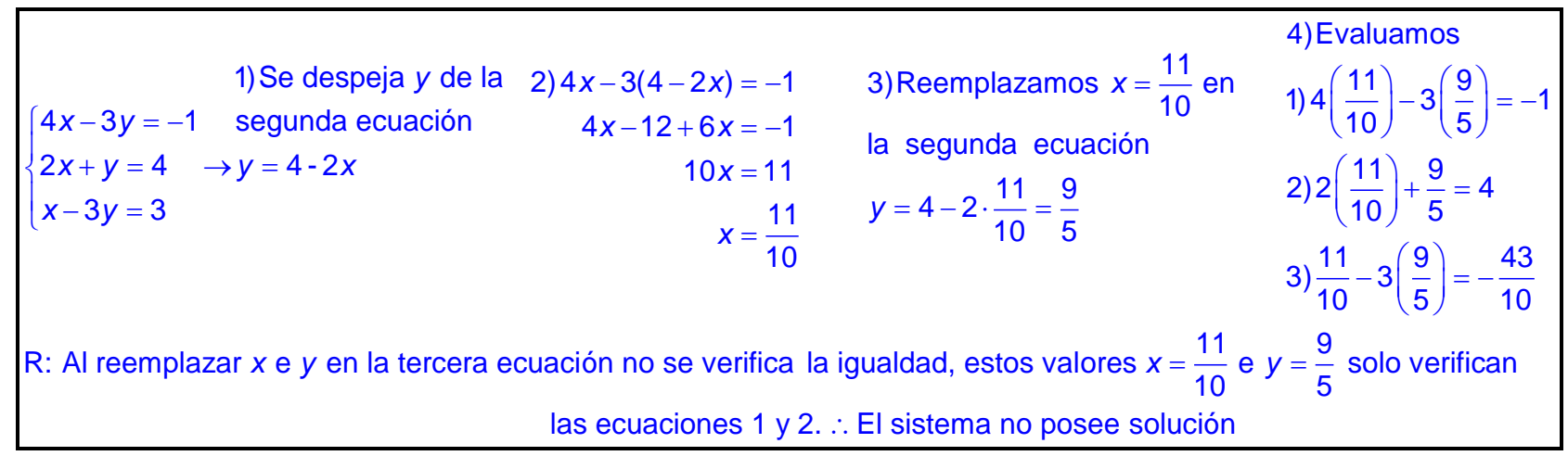

Fig. 2: Respuesta escrita del estudiante E2 a la tarea 2

Por otra parte, el estudiante E20 es uno de los que usó el método de reducción para resolver la tarea 2 (ver Fig. 3). Él etiquetó a las ecuaciones como 1, 2 y 3 . Luego, operó con las ecuaciones 2 y 3 de tal forma que eliminó la incógnita $y$, obteniendo un valor para la incógnita $x$. Por consiguiente, reemplazó el valor obtenido de $x$ en la ecuación 3 de donde obtuvo un valor para la incógnita $y$. Además, sustituyó el valor obtenido de $x$ en las ecuaciones 1 y 2 obteniendo otros valores para $y$. A partir de este proceso, él concluyo que el SEL no tiene solución, ya que los valores de y no coinciden en todas las rectas. Aquí, inferimos que E20 reconoce que un SEL tiene solución si los valores obtenidos para las incógnitas coinciden, independiente de la ecuación seleccionada, aunque él no habla de ecuaciones sino de rectas. Esto puede ser porque las ecuaciones del SEL dado corresponden gráficamente a rectas.

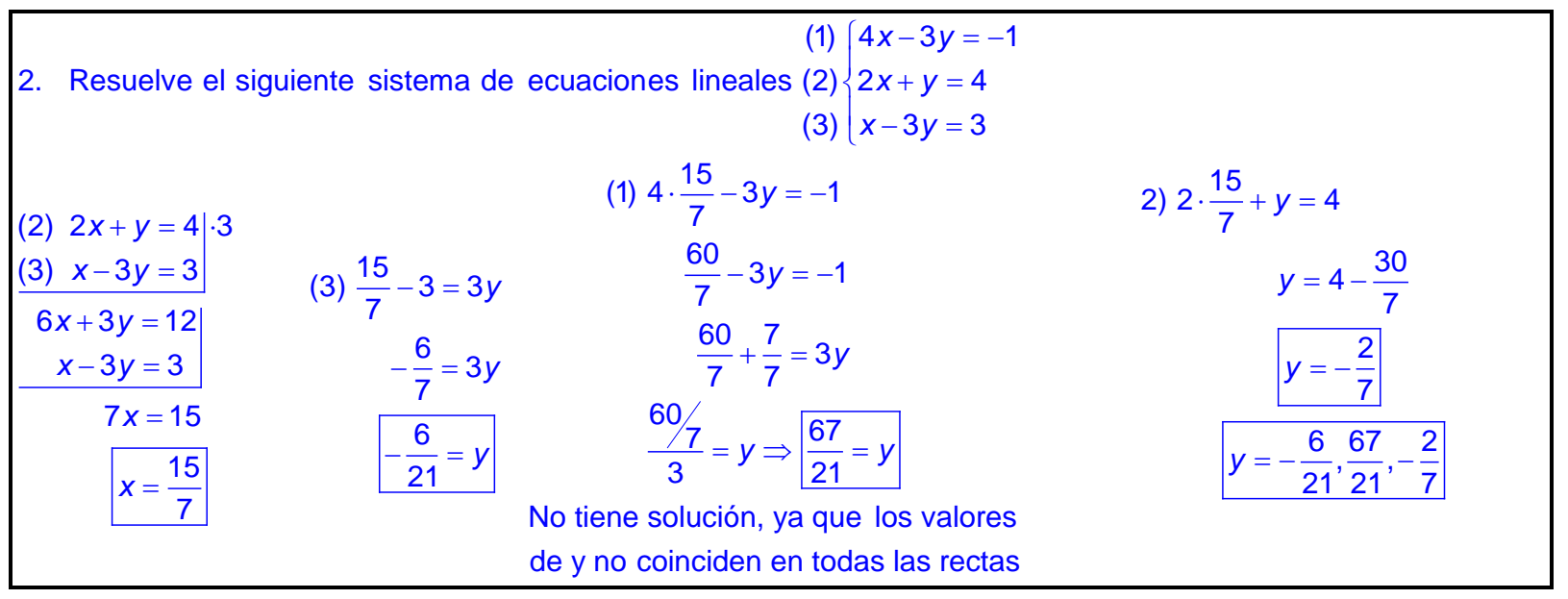

Fig. 3: Respuesta escrita del estudiante E20 a la tarea 2

Cabe señalar que solo once de los 60 estudiantes identifican que el SEL de $3 \times 2$ no tiene solución para las tareas 1 y 2. Por otra parte, se observa que al momento de justificar porqué el SEL de la tarea 1 no tiene solución, los estudiantes recurren a sus concepciones previas de representación gráfica de un SEL que contiene ecuaciones de rectas y el significado de la solución de un SEL de modo gráfico. En tanto, para justificar porqué el SEL de la tarea 2 no tiene solución, los estudiantes acuden a sus concepciones previas de resolución de SEL (método de sustitución, método de reducción o método de igualación). Lo anterior, permite inferir que la resolución de SEL en la enseñanza secundaria se enfoca, principalmente, en aquellos que poseen solución única vinculándolos con su representación gráfica como intercepción de rectas y utilizando uno de los tres métodos algebraicos habituales de resolución. Esto provoca inconvenientes en la resolución de este tipo de SEL lo cual se manifiesta en el bajo número de estudiantes que contestó correctamente a las tareas 1 y 2.

\section{EI SEL de 3x2 tiene 3 soluciones}

La mayoría de los estudiantes (87\%) que respondió de forma errónea a la tarea 1 sobre el SEL de $3 \times 2$ (representado gráficamente) escribió que dicho SEL tenía 3 soluciones. Varios de los estudiantes, entre ellos E7, que respondieron que hay 3 soluciones, señalaron en su respuesta escrita que es porque se interceptan las rectas en 3 puntos. En la Tabla 3 se observan algunos ejemplos de este tipo de respuestas de los estudiantes E7, E31 y E42. El estudiante E31 precisó que las soluciones de un SEL son representadas gráficamente como los puntos donde las rectas de cada ecuación se interceptan. En la figura se interceptan en 3 puntos, por lo tanto, tiene 3 soluciones. E31 muestra una concepción errónea sobre la solución de un 
SEL que está representado gráficamente, pues para él cada intersección de rectas es una solución, lo cual es cierto para un SEL de $2 \times 2$, pero no lo es para un SEL de $3 \times 2$ que corresponde al de esta tarea. Por otra parte, el estudiante E42 anotó lo mismo que E7, pero, además, agregó que las soluciones salen de resolver los 3 sistemas de $2 \times 2$ que se forman. De esta última afirmación de E42, podemos inferir que él tiene una concepción errónea de lo qué es la solución de un SEL porque cree que los 3 puntos, que se obtienen de resolver los 3 SEL de $2 \times 2$ que se forman con las 3 ecuaciones dadas, son soluciones del SEL de la tarea 2. Sin embargo, la solución de SEL debe satisfacer todas las ecuaciones de este al mismo tiempo.

De los estudiantes que respondieron que hay 3 soluciones, varios de ellos argumentaron en su respuesta escrita que en el gráfico hay 3 intersecciones, Por ejemplo, E17 dio esta respuesta y E3 escribió una respuesta similar a E17 (ver Tabla 3) precisando que se observan 3 intersecciones de las rectas correspondiente a cada solución. Estos estudiantes muestran una concepción errónea sobre la solución de un SEL de 3x2 que está representado gráficamente, pues para ellos cada intersección entre rectas es una solución del SEL. Los estudiantes que responden que el SEL de $3 \times 2$ de la tarea 1 (representado gráficamente) tiene 3 soluciones dan evidencia de que poseen una concepción errónea sobre cómo se representa la solución de una SEL de forma gráfica.

Tabla 3: Ejemplos de respuesta de los estudiantes que indicaron que el SEL de 3X2 de la Tarea tiene 3 soluciones

\begin{tabular}{|l|l|}
\hline Estudiante & Fragmento de respuesta \\
\hline E7 & El sistema tiene tres soluciones, ya que las rectas se interceptan en tres puntos. \\
\hline E31 & $\begin{array}{l}\text { Tiene tres soluciones, porque las soluciones son representadas gráficamente como los puntos donde } \\
\text { las rectas de cada ecuación se interceptan, en la figura se interceptan en tres puntos, por lo tanto, tiene } \\
\text { tres soluciones, }\end{array}$ \\
\hline E42 & $\begin{array}{l}\text { Tiene tres soluciones, dado que las tres rectas se interceptan en tres puntos. } \\
\text { Las soluciones salen de resolver los tres sistemas de 2x2 que se forman. }\end{array}$ \\
\hline E17 & El sistema tiene tres soluciones ya que hay tres intersecciones. \\
\hline E3 & $\begin{array}{l}\text { El sistema tiene tres soluciones, ya que gráficamente se observan tres intersecciones de las rectas } \\
\text { correspondiente a cada solución }\end{array}$ \\
\hline
\end{tabular}

\section{EI SEL de 3x2 tiene solución única}

De los estudiantes que responden de forma errónea a la tarea 2, el $47 \%$ concluye que el SEL de $3 \times 2$ (representado analíticamente) tiene solución única mientras que un $22 \%$ señala que tiene 3 soluciones y los demás, hacen un proceso, pero no concluyen el tipo de solución del SEL dado. Algunos estudiantes concluyeron que el SEL de $3 \times 2$ de la tarea 2, tiene solución única, usando como procedimiento inicial el método de igualación como se observa en la respuesta escrita del estudiante E17 en la Fig. 4 . E17 despeja $3 y$ de la primera ecuación del SEL $(4 x-3 y=-1)$ y de la tercera $(x-3 y=3)$. Luego, iguala las expresiones obtenidas de donde consigue un valor particular para $x$. A continuación, en la segunda ecuación del SEL $(2 x+y=4)$ reemplaza el valor de $x$ obtenido, y de esta forma, encuentra un valor particular para $y$. Así, E17 concluye que los valores de $x$ e y corresponden a: $x=-4 / 3$ e $y=20 / 3$. En este proceso de resolución de $E 17$ se evidencia que utiliza sus concepciones previas sobre resolución de un SEL representado en forma analítica. Sin embargo, él no verifica que los valores particulares obtenidos para $x$ e $y$ satisfagan cada una de las ecuaciones del SEL dado. De esto, se puede inferir que el estudiante E17 no tiene una comprensión profunda de lo qué es la solución de un SEL.

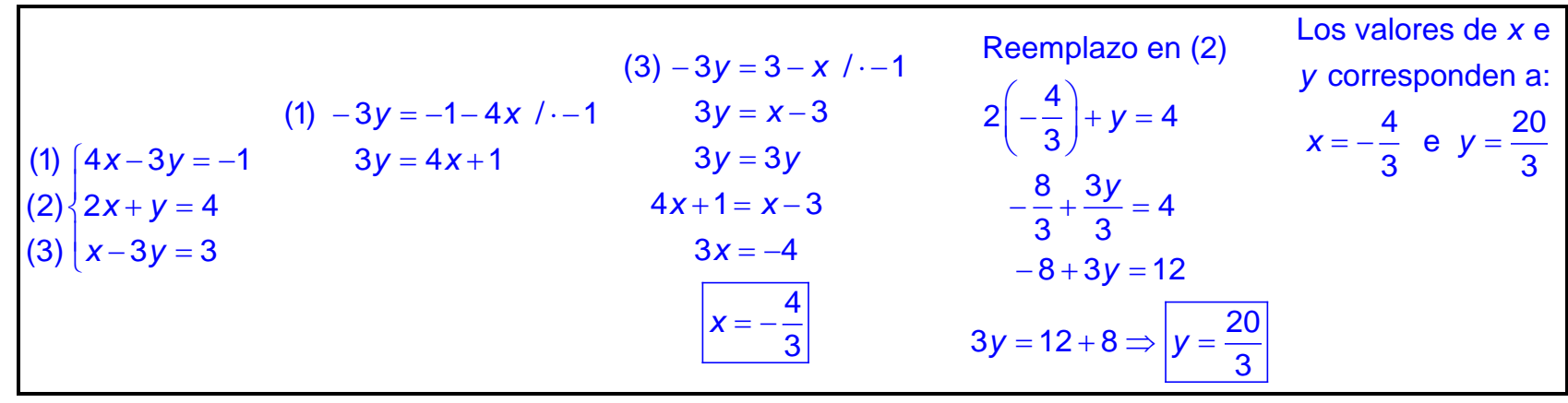

Fig. 4: Respuesta escrita de estudiante E17 a la tarea 2

Por otra parte, varios estudiantes concluyeron que el SEL de $3 \times 2$ de la tarea 2, tiene solución única, usando como procedimiento el tomar dos de las tres ecuaciones del SEL dado. Un ejemplo de esto, se observa en la 
respuesta escrita del estudiante E9 en la Fig. 5 . El estudiante E9 considera la primera y tercera ecuación del SEL $(x-3 y=-1$ y $x-3 y=3)$. Luego, utiliza el método de reducción con la finalidad de eliminar la incógnita $y$. A partir de esto, obtiene un valor particular para $x$. A continuación, toma ese valor de $x$ y lo reemplaza en la tercera ecuación del SEL. De aquí, obtiene un valor particular para $y$. Se infiere que la solución dada por el estudiante es $x=-4 / 3$ e $y=-7 / 9$ porque estos valores los encierra en un recuadro. En la respuesta escrita de E9, se observa que no verifica si los valores para $x$ e $y$ encontrados satisfacen la ecuación que no fue considerada, es decir, la tercera ecuación lineal dada en el SEL.

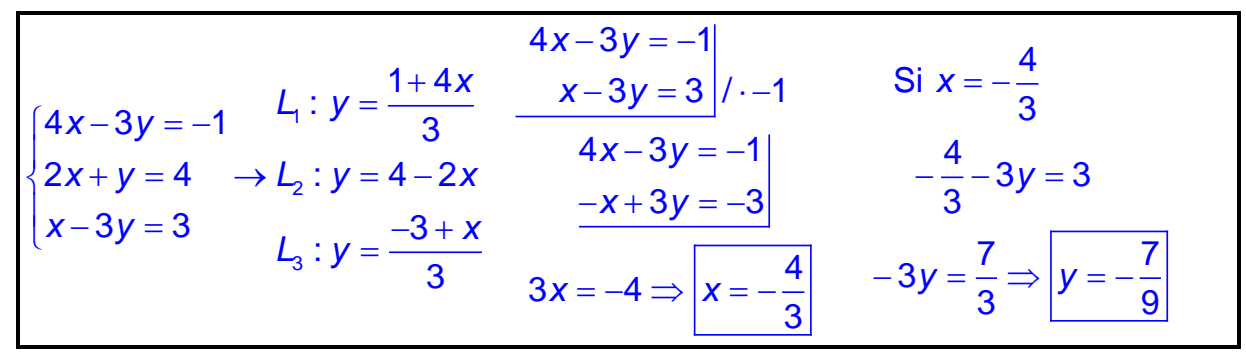

Fig. 5: Respuesta escrita de estudiante E9 a la tarea 2

Los estudiantes que concluyen de forma errónea que el SEL tiene solución única es porque no han comprendido en su totalidad a qué corresponde la solución de un SEL. Por esta razón, ellos solo se interesan por buscar valores para las incógnitas que se obtengan de al menos dos de las ecuaciones del SEL dado. Sin embargo, no se cuestionan si sería necesario considerar la tercera ecuación.

\section{DISCUSION FINAL}

Este estudio identificó las concepciones que muestran estudiantes de primer año de ingeniería cuando resuelven SEL de $3 \times 2$ que poseen solución vacía. En concreto, se identificaron tres concepciones de los estudiantes cuando resolvieron SEL de 3x2 que poseían solución vacía: el SEL de 3x2 no tiene solución, el SEL de $3 \times 2$ tiene 3 soluciones y el SEL de $3 \times 2$ tiene solución única. Las concepciones que predominan en los estudiantes al momento de resolver este tipo de SEL son las concepciones erróneas. Los estudiantes que responden que el SEL no tenía solución observaron en la tarea 1que no hay un único punto de intersección de las tres rectas. En tanto, los estudiantes que respondieron que el SEL de la tarea 2 no tenía solución escribieron que los valores para las incógnitas no satisfacían todas las ecuaciones del SEL dado.

En el caso de los estudiantes que indicaron que el SEL de $3 \times 2$ (representado de forma gráfica) tenía 3 soluciones, ya sea porque las rectas se interceptaban en 3 puntos o porque había 3 intercepciones, se infiere que ellos no sabían resolver un SEL por el método gráfico ni tampoco qué significaba la solución de un SEL. Esto puede ser porque en la enseñanza secundaria predomina el estudio de los métodos de resolución analítico. Otra razón, de esta concepción de los estudiantes es que, de acuerdo con Oktaç (2018), ellos generalizan la solución de un SEL, geométricamente, con la intersección de dos rectas en el plano. Por ende, como en el SEL dado en la tarea 1, se observan 3 intercepciones, concluyen que el SEL tiene 3 soluciones. En tanto, los estudiantes que escribieron que el SEL de 3x2 (representado de forma analítica) tenía solución única, en general, realizan un proceso de resolución en donde consideraron solo dos de las tres ecuaciones del SEL para determinar la solución de este. Este resultado coincide con lo encontrado por Almouloud y Bianchini (1996), quienes identificaron que, para resolver un SEL de 3x2, los estudiantes creen que son necesarias solo dos ecuaciones e ignoran el hecho de que la solución debe satisfacer todas las ecuaciones.

Concordamos con Amawa et al. (2019) que uno de los factores clave en el éxito de los estudiantes en la comprensión de un concepto matemático está determinado por el material que proporciona el docente. Este debe considerar, entre otros aspectos, los estudios sobre la enseñanza y aprendizaje del concepto. Por esto, sugerimos que el docente al momento de planificar y diseñar el material de aprendizaje sobre los SEL de $3 \times 2$ u otros tipos de SEL considere los resultados de nuestra investigación cautelando que los estudiantes construyan: las diferentes formas de representar un SEL, los métodos de resolución y los posibles tipos de solución. También, proponemos que el docente oriente el aprendizaje de los SEL por medio de las preguntas planteadas por Andrews-Larson (2015): ¿Cómo podemos resolver SEL?, ¿Qué significa ser una solución para un SEL? y ¿Cómo afecta la relación entre el número de incógnitas y el número de ecuaciones a la solución a un SEL? Además, con respecto a las diferentes formas de representación de un SEL, el docente podría apoyarse de tecnología como se sugiere en el trabajo de Mallet (2007).

Por otro lado, una limitación de este estudio es el número reducido de preguntas sobre SEL de 3x2. Para profundizar sobre este tipo de SEL, sería conveniente elaborar una evaluación que contemple SEL de 3x2 con los distintos tipos de soluciones de los SEL y no solo solución vacía como lo fue en este estudio. 


\section{CONCLUSIONES}

De acuerdo a los resultados obtenidos en este estudio exploratorio, se pueden plantear las siguientes conclusiones principales:

1.- Al presentar un SEL de 3x2 gráficamente (tarea 1), los estudiantes de esta investigación, movilizaron su modo de pensamiento sintético-geométrico. Además, inferimos que la mayoría de ellos, inmediatamente, activaron su concepción previa referente a que la intersección entre un par de rectas representa la solución de un SEL. Esto, consideramos que provocó en estos estudiantes una respuesta intuitiva y definitiva: el SEL tiene 3 soluciones debido a que hay 3 intersecciones.

2.- Al presentar un SEL de 3x2 de forma analítica (tarea 2), los estudiantes de esta investigación, movilizaron solo su modo de pensamiento analítico. De esto se infiere que ellos no hacen una conexión entre los modos de pensamiento sintético y analítico. Lo anterior, quizás porque en la enseñanza secundaria chilena se enfocan en resolver SEL, principalmente, a través de métodos algorítmicos.

3.- Las concepciones erróneas mostradas por los estudiantes al resolver un SEL de 3x2 con solución vacía dan evidencia que se necesita plantearle, a este tipo de estudiantes, tareas sobre SEL que promuevan el tránsito por los distintos modos de pensamiento en Álgebra Lineal (Sierpinska, 2000). Esto con la finalidad de minimizar sus dificultades con los SEL y en particular, con el significado de la solución de este.

\section{AGRADECIMIENTOS}

Este trabajo desarrollado gracias al apoyo del proyecto Fondecyt №11190284 y la colaboración de la Facultad de Ciencias de la Ingeniería de la Universidad Austral de Chile.

\section{REFERENCIAS}

Andrews-Larson, C. Roots of linear algebra: An historical exploration of linear systems, https://doi.org/10.1080/10511970.2015.1027975, Primus, 25(6), 507-528, (2015).

Almouloud, S. A., y Bianchini, B. L. O erro ligado ao ensino/aprendizagem de sistemas lineares, Anais do IV Encontro Paulista de Educação Matemática por R. C. Lins, pp 216-223, Sociedade Brasileira de Educação Matemática, São Paulo, Brasil (1996).

Amawa, I. M., Yerizon, Y., Sri, N., y Putra, R. T. Development of students' worksheet based on apos theory approach to improve student achievement in learning system of linear equations, Int. J. of Sci. Tech. Res., ISSN: 2277-8616, 8(4), 287292 (2019).

Maxwell, J. A. Understanding and validity in qualitative research, https://doi.org/10.17763/haer.62.3.8323320856251826, Harv. Educ. Rev., 62(3), 279-301 (1992).

MINEDUC. Bases Curriculares $7^{\circ}$ básico a $2^{\circ}$ medio. Ministerio de Educación de Chile. Santiago, Chile (2015)

Mallet, D. G. Multiple representations for systems of linear equations via the computer algebra system Maple, Int. Elec. J. Math. Educ., ISSN: 1306-3030, 2(1), 16-31 (2007).

Oktaç, A. Conceptions About System of Linear Equations and Solution, Challenges and Strategies in Teaching Linear Algebra; In ICME-13 Monograph by S. Stewart, C. Andrews-Larson, A. Berman, y M. Zandieh, pp 71-101. Springer, Cham, Alemania (2018).

Peña, A. Análisis de los errores y dificultades en la solución de sistemas de ecuaciones lineales en estudiantes de ingeniería, Tesis de magister, Pontificia universidad católica del Perú, Perú (2019)

Possani, E., Trigueros, M., Preciado, J. G., y Lozano, M. D. Use of models in the teaching of linear algebra, https://doi.org/10.1016/j.laa.2009.05.004, Linear Algebra Appl., 432(8), 2125-2140, (2010).

Roa-Fuentes, S., y Parraguez, M. Estructuras mentales que modelan el aprendizaje de un teorema del álgebra lineal: Un estudio de casos en el contexto universitario, http://dx.doi.org/10.4067/S0718-50062017000400003, Form. Univ., 10(4), 15-32, (2017).

Rodríguez M. A., Mena A., Mena J.J., Vásquez P., y Del Valle M.E. Construcción cognitiva del conjunto solución de un sistema de ecuaciones lineales con dos incógnitas, https://doi.org/10.5565/rev/ensciencias.2194, Enseñ. Cienc. Rev. Invest. Exp. Didact., 37(1), 71-92 (2019)

Sierpinska, A. On some aspects of students' thinking in linear algebra; In On the teaching of linear algebra by J.L. Dorier, vol 23, pp 209-246, Springer, Dordrecht, Países Bajos (2000)

Zandieh, M., y Andrews-Larson, C. Symbolizing while solving linear systems, https://doi.org/10.1007/s11858-019-010833, ZDM, 51(7), 1183-1197 (2019). 